\title{
Electromagnetic Solutions of the Field Equations in Presence of Zeromass Mesons
}

\author{
R. M. Misra and Deo Bhushan Pandey \\ Department of Physics, University of Gorakhpur, Gorakhpur, India
}

Received November 4, 1970

\begin{abstract}
The field equations of general relativity with electromagnetic stress tensor and zeromass scalar meson field are investigated. The metric coefficients are assumed to be functions of three variables only. It is then shown that, if one assumes a functional relation between some one of the metric coefficients and the electromagnetic potentials, that one can find a solution of the coupled Einstein-Maxwell equations in terms of a solution of the Einstein equations with zeromass scalar meson field as source.
\end{abstract}

\section{Introduction}

In this note we have investigated the nonempty field equations of general relativity where the stress-energy tensor of electrodynamics and zeromass meson field is taken as the source. Although the physical implications to be drawn from the study of zeromass meson fields suffer from the lack of any experimental support, nevertheless, it has been shown by Janis et al. [1] that a zeromass meson field interacting with a static, spherically symmetric and asymptotically flat gravitational field has some very interesting and surprising features. We have also investigated, elsewhere, the interaction of zeromass meson field with charged incoherent matter in a stationary axially symmetric gravitational field and have found that the spacetime is basically modified and leads to some interesting consequences [2]. Further, exact solutions of the field equations are scarce, and it is always worthwhile to obtain analytic solutions of a problem with more than one field coupled nonlinearly.

However, we shall not derive here any explicit solutions of the field equations corresponding to the problem stated above, rather, we shall discuss a procedure which will enable one to construct exact electromagnetic solutions from a given solution of the field equations with massless scalar meson field as source. It has been shown by Harrison [3] and others $[4,5]$ that one can generate a solution of the coupled EinsteinMaxwell equations with a nonvanishing electromagnetic field from a given vacuum solution of the field equations in which the metric coefficients are functions of not more than three variables. This result is a 
consequence of the assumption of functional dependence between some of the metric coefficients and the electromagnetic potentials. Several authors have made use of this functional relationship for generating solutions of the field equations [6]. In this note we present a theorem which may be considered in a sense, a generalisation of the previous result referred to above. We assume that the metric coefficients $g_{i j}$, electromagnetic field $F_{i j}$ and the scalar meson field $\phi$ are functions of $x^{1}, x^{2}$ and $x^{3}$ only. It is then shown that if one assumes a functional relationship between $g_{00}$ and the electromagnetic potentials that one can find a solution of the coupled gravitational-electromagnetic equations in terms of a solution of the field equations with massless scalar field as source.

In Section 2 we obtain the Maxwell and Einstein equations in suitable form by assuming a functional relationship between $g_{00}$ and electromagnetic potentials. The desired result is obtained in Section 3. The last section contains some concluding remarks.

\section{The Field Equations}

The equations under consideration are ${ }^{1}$

$$
\begin{gathered}
G_{i j} \equiv R_{i j}-\frac{1}{2} g_{i j} R=-8 \pi T_{i j}, \\
F_{; j}^{i j}=0, \\
F_{[i j ; k]}=0, \\
\phi_{; i}^{i}=0
\end{gathered}
$$

with

and

$$
T_{i j}=M_{i j}+f_{i j}
$$

where

$$
\phi_{i}=\phi_{, i}
$$

1 The range of Latin indices is from 0 to 3 whereas Greek indices vary from 1 to 3 . unless otherwise stated. Summation convention is assumed throughout. Comma and semicolon followed by a suffix denote ordinary and covariant differentiation, respectively. [] is the symbol for skewsymmetrisation. 
Further, we choose the line element in the following form

$$
d s^{2}=e^{2 U}\left(d x^{0}\right)^{2}+e^{-2 U} \gamma_{\alpha \beta} d x^{\alpha} d x^{\beta} .
$$

This metric is the general static line element and admits a hypersurface orthogonal Killing vector. The three dimensional metric $\gamma_{\alpha \beta}$ satisfies the following

$$
\gamma_{\alpha \beta} \gamma^{\alpha \lambda}=\delta_{\beta}^{\lambda} .
$$

The nonvanishing components of the Ricci tensor for the metric (8) are given by

$$
\begin{gathered}
R_{00}=e^{4 U} \Delta_{2}(U), \\
R_{0 \alpha}=0 \\
R_{\alpha \beta}=P_{\alpha \beta}+2 U_{, \alpha} U_{, \beta}-\gamma_{\alpha \beta} \Delta_{2}(U)
\end{gathered}
$$

where we have introduced differential parameters of first and second order defined as [7].

$$
\begin{gathered}
\Delta_{1}(U)=\gamma^{\alpha \beta} U_{, \alpha} U_{, \beta}, \\
\Delta_{1}(U, V)=\gamma^{\alpha \beta} U_{, \alpha} V_{, \beta}, \\
\Delta_{2}(U)=\gamma^{\alpha \beta} U_{; \alpha \beta}=\gamma^{\alpha \beta}\left(U_{, \alpha \beta}-\Sigma_{\alpha \beta}^{\lambda} U_{, \lambda}\right) .
\end{gathered}
$$

$\sum_{\alpha \beta}^{\lambda}$ and $P_{\alpha \beta}$ are the Christoffel symbols and Ricci tensor, respectively, defined with respect to $\gamma_{\alpha \beta}$.

Next we consider Maxwell Eqs. (2) and (3) which may be expressed in the following form

$$
\begin{aligned}
{\left[(-g)^{\frac{1}{2}} F^{0 \alpha}\right]_{, \alpha} } & =0, \\
{\left[(-g)^{\frac{1}{2}} F^{\beta \alpha}\right]_{, \alpha} } & =0, \\
F_{\alpha 0, \beta}+F_{0 \beta, \alpha} & =0, \\
\varepsilon^{\alpha \beta \gamma} F_{\alpha \beta, \gamma} & =0
\end{aligned}
$$

where $\varepsilon^{\alpha \beta \gamma}$ is 3 -index indicator, taking values +1 or -1 if $\alpha, \beta, \gamma$ are even or odd permutations of 1,2 and 3 , respectively, and zero otherwise. If one now defines the potentials $A$ and $B$ in the following manner

$$
\begin{aligned}
& F^{\alpha \beta}=(-g)^{-\frac{1}{2}} \varepsilon^{\alpha \beta \lambda} A_{, \lambda}, \\
& F_{0 \alpha}=B_{, \alpha} .
\end{aligned}
$$


Eqs. (17) and (18) are identically satisfied. Further making use of Eqs. (13), (14), (15), (20), and (21) in (16) and (19) we get

$$
\begin{aligned}
& \Delta_{2}(A)-2 \Delta_{1}(U, A)=0, \\
& \Delta_{2}(B)-2 \Delta_{1}(U, B)=0 .
\end{aligned}
$$

We now obtain the components of $f_{i j}$. We get

$$
\begin{gathered}
f_{00}=\frac{1}{8 \pi} e^{2 U}\left(\Delta_{1}(A)+\Delta_{1}(B)\right), \\
f_{0 \alpha}=0, \\
f_{\alpha \beta}=\frac{1}{8 \pi} e^{-2 U}\left[2\left(A_{, \alpha} A_{, \beta}+B_{, \alpha} B_{, \beta}\right)-\gamma_{\alpha \beta}\left(\Delta_{1}(A)+\Delta_{1}(B)\right)\right] .
\end{gathered}
$$

The symmetrical form of Eqs. (22), (23), (24a), and (26a) with respect to potentials $A$ and $B$ enables one to introduce a new potential $C$ defined as [8]

$$
\begin{aligned}
& A=C \operatorname{Cos} \alpha, \\
& B=C \operatorname{Sin} \alpha
\end{aligned}
$$

$\alpha$ being a constant. In view of (27) we may write Eqs. (22) and (23) as a single equation

$$
\Delta_{2}(C)-2 \Delta_{1}(U, C)=0
$$

Further, the components of $f_{i j}$ assume the following form

$$
\begin{gathered}
f_{00}=\frac{1}{8 \pi} e^{2 U} \Delta_{1}(C), \\
f_{0 \alpha}=0, \\
f_{\alpha \beta}=\frac{e^{-2 U}}{8 \pi}\left(2 C_{. \alpha} C_{, \beta}-\gamma_{\alpha \beta} \Delta_{1}(C)\right) .
\end{gathered}
$$

In a similar manner, we may obtain the components of $M_{i j}$.

Now the field equations can easily be set up and one obtains

$$
\begin{gathered}
\Delta_{2}(U)=-e^{-2 U} \Delta_{1}(C), \\
P_{\alpha \beta}+2 U_{, \alpha} U_{, \beta}=-8 \pi \phi_{\alpha} \phi_{\beta}-2 e^{-2 U} C_{. \alpha} C_{, \beta}
\end{gathered}
$$

and Eqs. (28) and (4). 


\section{Derivation of the Electromagnetic Solutions}

We now assume that $U$ and $C$ are functionally related i.e.

$$
U=U(\theta), \quad C=C(\theta) .
$$

However, a more manageable form of the field equations results if we write

$$
V=e^{U} .
$$

As a result of this transformation, we obtain

$$
\begin{aligned}
\Delta_{2}(U) & =\frac{1}{V} \Delta_{2}(V)-\frac{1}{V^{2}} \Delta_{1}(V), \\
\Delta_{1}(U, C) & =\frac{1}{V} \Delta_{1}(V, C) .
\end{aligned}
$$

Hence the Eqs. (28a)-(30a) are obtained as

$$
\begin{gathered}
\Delta_{2}(C)-\frac{2}{V} \Delta_{1}(V, C)=0, \\
\Delta_{2}(V)-\frac{1}{V} \Delta_{1}(V)=-\frac{1}{V} \Delta_{1}(C), \\
P_{\alpha \beta}+\frac{2 V_{, \alpha} V_{, \beta}}{V^{2}}=-8 \pi \phi_{\alpha} \phi_{\beta}-\frac{2}{V^{2}} C_{, \alpha} C_{, \beta} .
\end{gathered}
$$

Now we use the following form of the functional dependence (31)

$$
V=V(\theta), \quad C=C(\theta)
$$

where $\theta=\theta\left(x^{1}, x^{2}, x^{3}\right)$. Thus, we obtain

$$
\begin{aligned}
\Delta_{1}(V) & =V^{\prime 2} \Delta_{1}(\theta), \\
\Delta_{1}(V, C) & =V^{\prime} C^{\prime} \Delta_{1}(\theta), \\
\Delta_{2}(V) & =V^{\prime} \Delta_{2}(\theta)+V^{\prime \prime} \Delta_{1}(\theta)
\end{aligned}
$$

and similar expressions with respect to $C$. Here an overhead prime indicates differentiation with respect to $\theta$. Substitution of the above expressions in Eqs. (28 b)-(30 b) yields

$$
\begin{gathered}
\Delta_{2}(\theta)+\left(\frac{C^{\prime \prime}}{C^{\prime}}-\frac{2 V^{\prime}}{V}\right) \Delta_{1}(\theta)=0, \\
\Delta_{2}(\theta)+\left(\frac{V^{\prime \prime}}{V^{\prime}}-\frac{V^{\prime 2}-C^{\prime 2}}{V V^{\prime}}\right) \Delta_{1}(\theta)=0, \\
P_{\alpha \beta}=-8 \pi \phi_{\alpha} \phi_{\beta}-\frac{2}{V^{2}}\left(C^{\prime 2}+V^{\prime 2}\right) \theta_{, \alpha} \theta_{, \beta} .
\end{gathered}
$$


If we now assume that $\Delta_{1}(\theta) \neq 0$, we observe that the Eqs. (34) and (35) become identical provided that

$$
\begin{aligned}
C^{\prime \prime} & =\frac{2 V^{\prime} C^{\prime}}{V}, \\
V^{\prime \prime} & =\frac{\left(V^{\prime 2}-C^{\prime 2}\right)}{V} .
\end{aligned}
$$

The solution of these equations may be taken as

$$
\begin{aligned}
& V=\lambda \operatorname{sech} \theta, \\
& C=\lambda \tanh \theta
\end{aligned}
$$

where $\lambda$ is a constant. In the case, thus, we have a linear differential equation for $\theta$ so that we may readily obtain a solution of Eqs. (28a) and (29a) with two arbitrary functions.

If we now substitute (39) and (40) in (36), we find the equations

$$
P_{\alpha \beta}=-8 \pi \phi_{\alpha} \phi_{\beta}-2 \theta_{, \alpha} \theta_{, \beta} .
$$

But if we took the field equations with meson field only i.e. $C=0$ and put $V=\exp (\theta)$ we would obtain Eqs. (4), (29a) with right hand side absent and (41) (of course in (29a) $U$ replaced by $\theta$ ). Thus we have established that

"For every solution $V=\exp (\theta)$ and $\gamma_{\alpha \beta}$ of the field equations with the energy tensor of a massless scalar meson as source we may also form a solution of the coupled Einstein-Maxwell equations with a nonvanishing electromagnetic field with the same $\gamma_{\alpha \beta}$ and the functions $V=\lambda \operatorname{sech} \theta$, $C=\tanh \theta^{\prime \prime}$.

This result may be stated in an alternative simple form. Suppose, the metric

$$
d s^{2}=V^{2} d t^{2}+V^{-2} \gamma_{\alpha \beta} d x^{\alpha} d x^{\beta}
$$

with $V$ and $\gamma_{\alpha \beta}$ functions of $x^{1}, x^{2}$ and $x^{3}$ satisfied the field equations with massless meson field as source. Then the metric

$$
d s^{2}=\frac{4 \lambda^{2} V^{2}}{\left(V^{2}+1\right)^{2}} d t^{2}+\frac{\left(V^{2}+1\right)^{2}}{4 \lambda^{2} V^{2}} \gamma_{\alpha \beta} d x^{\alpha} d x^{\beta}
$$

and the potentials

$$
C=\lambda \frac{\left(V^{2}-1\right)}{\left(V^{2}+1\right)}
$$

satisfy the Einstein-Maxwell equations. 
In the derivation of the above results we have assumed that all the functions are independent of $x^{0}$. However, the results are quite general and hold good with appropriate modifications when the absent coordinate may be any of the four variables. Further, we have also not assumed any functional relationship between the field $\phi$ and the metric coefficient. But if this is done, and $x^{3}$ is taken as the absent coordinate ${ }^{2}$ further interesting results are obtained. Obviously, in this case the gradient of $\theta$ may be taken as a null vector. In view of this fact and since $\phi$ and $\theta$ are functionally related, $M_{i j}$ turns out to be the energy-momentum tensor for the unidirectional flow of pure radiation. If, for the moment we assume that the electromagnetic field is absent, then it has been shown by Misra [9] that if $V=\exp (\theta)$ and $\gamma_{\alpha \beta}$ is a vacuum solution of the field equations, then $\exp (U)$ and $\gamma_{\alpha \beta}$ is a solution of the field equations for unidirectional flow of pure radiation i.e. for

where

$$
R_{i j}=\sigma \theta_{, i} \theta_{, j}
$$

and

$$
\sigma=2\left(U^{\prime 2}-1\right)
$$

$$
g^{i j} \theta_{, i} \theta_{, j}=0 \quad \text { and } \quad U=U(\theta) .
$$

We are now in a position to generalise this result.

For every vacuum solution $V=\exp (\theta)$ and $\gamma_{\alpha \beta}$ of the field equations, we may also form a solution of the pure radiation field equations with same $\gamma_{\alpha \beta}$ and $U=U(\theta)$ such that the density of the pure radiation is given by (46), and a solution of the coupled Einstein-Maxwell equations, with a nonvanishing electromagnetic field and the functions $V=\lambda \operatorname{sech} \theta$, $C=\lambda \tanh \theta$.

\section{Concluding Remarks}

Aside from the immediate utility of these investigations in generating electromagnetic solutions of the field equations, one may also use the results obtained here in discussing the singularities of the two fields. One may easily observe that, $\gamma_{\alpha \beta}$ being the same in both cases, the two spaces will have the same singularities if any, present in $\gamma_{\alpha \beta}$. Further, the same is also true for the singularities $V=0$ if $V^{2} \ll 1$. Thus we see that there is one to one correspondence between the singularities in both cases except when $V$ is large (since $V^{2} /\left(V^{2}+1\right)$ tends to zero).

In conclusion we hope that these results will lead to deeper understanding of gravito-electrodynamics.

${ }^{2}$ In fact the absent coordinate may be taken as any one of $x^{1}, x^{2}$ and $x^{3}$ and the results hold. 


\section{References}

1. Janis, A. I., Newman, E. T., Winicour, J.: Phys. Rev. Letters 20, 878 (1968).

2. Misra, R. M., Bhushan Pandey, Deo: Intraction of zeromass mesons and charged dust in axially symmetric fields (1970) (communicated).

3. Harrison, B. K.: Phys. Rev. 138, B 488 (1965).

4. Misra, M., Radhakrishna, L.: Proc. Nat. Inst. Sci. India A 28, 632 (1962).

5. Misra, R. M.: Phys. Rev. (1970). To appear.

6. Weyl, H.: Ann. Physik, 54, 117 (1917); Majumdar, S. D.: Phys. Rev. 72, 390 (1947); Bonnor, W. B.: Proc. Phys. Soc. A 66, 145 (1953) and references 3 and 4.

7. Eisenhart, L. P.: Riemannian geometry. Princeton, N. J.: Princeton University Press 1949.

8. This transformation may be looked upon as the "duality rotation" of Misner and Wheeler (Misner, C. W., Wheeler, J. A.: Ann. Phys. 2, 525 (1957)). We may take the electromagnetic field to be purely electric or purely magnetic by choosing $A$ or $B$ to be zero. respectively.

9. Misra, M.: Ann. Inst. Henri Poincaré 7, 245 (1967).

R. M. Misra

Deo Bhushan Pandey

Department of Physics

University of Gorakhpur

Gorakhpur (U.P.), India 
